\title{
Conexão cavopulmonar parcial como primeiro estágio no tratamento do coração univentricular em um centro na Amazônia: resultados imediatos
}

\author{
Initial aproach parcial cavopulmonary anastomosis in univentricular repair at a center in \\ the Amazon: Analysis of early outcomes
}

\section{Anastomosis cavopulmonar parcial de abordaje inicial en reparación univentricula en un centro en el Amazonas: análisis de resultados tempranos}

Camila Magno Sozinho Pereira ${ }^{1 *}$, Mauricio Fortuna Pinheiro ${ }^{1}$, Renan Kleber Costa Teixeira ${ }^{2}$, Kathia de Oliveira Harada1.

\section{RESUMO}

Objetivo: Analisar a eficácia da conexão cavopulmonar parcial como paliação primária no tratamento do coração univentricular em pacientes com mais de três meses. Métodos: Foram avaliados retrospectivamente os prontuários de pacientes portadores de cardiopatias congênitas com fisiologia univentricular submetidos à conexão cavopulmonar como primeiro estágio. Foram avaliados os seguintes parâmetros: idade, sexo, diagnóstico anatômico, cardiopatias associadas, tempo de circulação extracorpórea, tempo de intubação, tempo de internação hospitalar, complicações pós-operatórias, saturação periférica de oxigênio e da pressão sistólica da artéria pulmonar pré e pós-operatória. Resultados: Foram identificados 11 casos de crianças submetidas a conexão cavopulmonar parcial como paliação primária. A média de idade foi 10,54 meses, com leve prevalência do sexo feminino $(54,54 \%)$. As principais características anatômicas das cardiopatias foram Atresia tricúspide e Ventrículo Único tipo esquerdo. Houve aumento na saturação de oxigênio variando de uma média de $74,46 \%$ para $88,27 \%$ ( $p<0,01$ ), com adequação da pressão sistólica de artéria pulmonar de $20,80 \mathrm{mmHg}$ para $17,80 \mathrm{mmHg}(\mathrm{p}<0,05)$. Ocorreram dois casos de paresia frênica e um caso de quilotórax os quais foram corrigidos cirurgicamente. Não houve óbitos na amostra. Conclusão: A conexão cavopulmonar parcial como paliação primária no tratamento do coração univentricular teve resultados imediatos satisfatórios quando realizada após os três meses de idade.

Palavras-chave: Cardiopatias congênitas, Anormalidades congênitas, Canal arterial.

\begin{abstract}
Objective: To evaluate the efficacy of the bidirectional cavopulmonary connection as primary palliation in the treatment of the univentricular heart in patients older than three months. Methods: We retrospectively evaluated the medical records of patients with congenital heart defects with univentricular morphophysiology undergoing cavopulmonary connection as first stage. The following parameters were evaluated: age, gender, anatomical diagnosis, associated heart defects, time of extracorporeal circulation, intubation time, length of hospital stay and postoperative complications, variation of peripheral oxygen saturation and pre and postoperative pulmonary artery systolic pressure was evaluated. The main anatomical characteristics of the heart defects were tricuspid atresia and single ventricle left type. Results: Eleven cases of children submitted to a partial cavopulmonary connection as primary palliation were identified. The mean age was 10.54 months, with a slight prevalence of females $(54.54 \%)$. There was an increase in oxygen saturation ranging from a mean of $74.46 \%$ to $88.27 \%$ ( $p<0.01$ ), with pulmonary artery systolic pressure adjusting from $20.80 \mathrm{mmHg}$ to $17.80 \mathrm{mmHg}(p<0.05)$. There were two cases of phrenic paresis and one case of chylothorax which were corrected surgically. There were no deaths in the sample. Conclusion: Initial approach in univentricular repair is safe and showed reasonable early outcomes when performed after 3 months of age.
\end{abstract}

Keywords: Congenital heart diseases, Congenital abnormalities, Ductus arteriosus.

${ }^{1}$ Fundação Hospital de Clínicas Gaspar Vianna (FHCGV), Belém-PA. *E-mail: camilasozinho@yahoo.com.br 2Universidade do Estado do Pará (UEPA). Belém-PA. 


\section{RESUMEN}

Objetivo: Analizar la eficacia de la conexión cavopulmonar parcial como paliativo primario en el tratamiento del corazón univentricular en pacientes mayores de tres meses. Método: Evaluamos retrospectivamente los registros médicos de pacientes con cardiopatía congénita con fisiología univentricular que se sometieron a una conexión cavopulmonar como primera etapa. Se evaluaron los siguientes parámetros: edad, sexo, diagnóstico anatómico, enfermedad cardíaca asociada, tiempo de derivación cardiopulmonar, tiempo de intubación, duración de la estancia hospitalaria, complicaciones postoperatorias, saturación periférica de oxígeno y presión sistólica de la arteria pulmonar pre y posoperatoria. operativo Resultados: Once casos de niños que se sometieron a una conexión cavopulmonar parcial se identificaron como paliación primaria. La edad promedio fue de 10.54 meses, con una ligera prevalencia femenina (54.54\%). Las principales características anatómicas de las enfermedades cardíacas fueron la atresia tricúspide y el ventrículo izquierdo único. La saturación de oxígeno aumentó de un promedio de $74.46 \%$ a $88.27 \%(p<0.01)$, con una presión sistólica de la arteria pulmonar adecuada de $20.80 \mathrm{mmHg}$ a $17.80 \mathrm{mmHg}(p<0.05)$.) Hubo dos casos de paresia frénica y un caso de quilotórax que fueron corregidos quirúrgicamente. No hubo muertes en la muestra. Conclusión: La conexión cavopulmonar parcial como paliación primaria en el tratamiento del corazón univentricular tuvo resultados inmediatos satisfactorios cuando se realizó después de los tres meses de edad.

Palabras clave: Defectos cardíacos, Congénitos, Anomalías congénitas, Ductus arterioso.

\section{INTRODUÇÃO}

Cardiopatia congênita é uma anormalidade na estrutura ou função cardiocirculatória, ocorrente desde o nascimento, mesmo que diagnosticada posteriormente. Pode resultar em morte intraútero, na infância ou na idade adulta. Foi responsável por 6\% dos óbitos infantis, no Brasil, em 2007 (PINTO VC, et al., 2015).

Uma metanálise sobre a prevalência global das cardiopatias congênitas que incluiu 114 estudos, com uma população de 24.091 .867 nascimentos demonstrou que a taxa de prevalência estimada de 9,1 cardiopatias congênitas para cada 1.000 nascimentos permanece estável nos últimos 15 anos. Isso corresponde a 1,35 milhão de recém-nascidos com cardiopatias congênitas cada ano (VAN DER LINDE D, et al, 2011).

Variam em gravidade, ocorrendo desde comunicações entre as cavidades cardíacas que podem até regredir espontaneamente até as malformações complexas, como o Coração Univentricular, que requerem vários procedimentos cirúrgicos e ou hemodinâmicos. Clinicamente manifestam-se principalmente de sob três formas: cianose, arritmia ou insuficiência cardíaca (dispneia as mamadas, ganho ponderal deficiente, infecções respiratórias de repetição) (GO AS, et al., 2013).

No âmbito das cardiopatias congênitas complexas, o manuseio cirúrgico do coração univentricular corresponde a estratégia desafiadora, associada a elevado risco de mortalidade consequente a baixa expectativa de correção anatômica. Neste sentido resta a opção de tratamento da hipóxia (correção fisiológica), onde o fluxo sanguíneo arterial pulmonar é desconectado cirurgicamente de sua via anterógrada ventricular, pulsátil e natural, através do redirecionamento de fluxo do retorno das veias sistêmicas do coração para as artérias pulmonares, denominada cirurgia de conexão cavopulmonar parcial ou Glenn bidirecional (BG) (HAUCK A, et al., 2017; ZAHR RA, et al., 2016).

Considerando a superfície corpórea e vasos ainda pequenos, bem como a elevada resistência vascular pulmonar do recém-nascido, a conexão cavopulmonar não é realizada no período neonatal, fazendo-se necessário o estadiamento cirúrgico da doença, através da realização de 3 cirurgias cardíacas até alcançar o desvio sanguíneo venoso sistêmico completo (HAUCK A, et al., 2017; ZAHR RA, et al., 2017).

$\mathrm{Na}$ fase neonatal, quando a manifestação clínica de hipóxia é severa e na impossibilidade de realização de conexão cavopulmonar parcial, faz-se necessário a confecção de um shunt sistêmico pulmonar (SASIKUMAR N, et al., 2017). 
Em contrapartida, existem circunstâncias onde não há restrição ao fluxo sanguíneo pulmonar e a insuficiência cardíaca é preponderante, sendo necessária realização da técnica de cerclagem da artéria pulmonar (SASIKUMAR N, et al., 2014).

Posteriormente, após ganho de estatura, peso e sobretudo quando as pressões de artéria pulmonar estão em níveis mais baixos, normalmente entre os 6 e 12 meses de idade, indica-se a correção cirúrgica fisiológica da hipoxemia: a conexão cavopulmonar parcial ou cirurgia de Glenn Bidirecional (BG) e habitualmente entre 1 e 5 anos de idade a última intervenção, a conexão cavopulmonar total ou cirurgia de Fontan (HAUCK A, et al., 2017; ZAHR RA, et al., 2016).

Apesar de estes procedimentos fazerem parte do estadiamento cirúrgico padronizado na maioria dos centros de cirurgia cardiopediátrica, sabe-se que a mortalidade se mostra mais elevada entre o primeiro e 0 segunda estágio cirúrgico, ou seja, antes da confecção da derivação cavopulmonar parcial (BG) (HAUCK A, et al., 2017; SASIKUMAR N, et al.,2014).

Não obstante, identifica-se ao nascimento que algumas variações anatômicas podem apresentar um certo balanço de fluxos entre a circulação sistêmica e pulmonar, com níveis de hipoxemia toleráveis, sem sinais exuberantes de insuficiência cardíaca, permitindo que evoluam com ganho ponderal satisfatório. Nestes casos opta-se pela vigilância clínica observacional até idade ideal para realizar-se a conexão cavopulmonar parcial (BG) (HAUCK A, et al., 2017; ZAHR RA, et al., 2016).

Em contrapartida, condições socioeconômicas adversas no Brasil, as quais supõe-se restringir o diagnóstico pré e pós-natal, propiciam atendimentos e diagnósticos tardios, onde o planejamento terapêutico não foi realizado e eventualmente a descompensação cardíaca e risco de morte são imprevisíveis (TANOUE Y, et al.,2007).

Ademais, o centro de coleta de dados deste estudo é o único centro de referência em cardiopediatria de nosso estado, onde muitos pacientes são atendidos tardiamente e em caráter emergencial para a primeira cirurgia cardíaca com corações univentriculares. Portanto surge o questionamento para esta pesquisa: É seguro a abordagem com conexão cavopulmonar parcial (BG) como tratamento cirúrgico inicial na condição de hipóxia e coração univentricular?

Sendo assim, o objetivo deste estudo foi analisar a eficácia da cirurgia de conexão cavopulmonar parcial (BG) como paliação primária no tratamento do coração univentricular, através avaliação da saturação de oxigênio e pressão de artéria pulmonar, bem como os aspectos clínicos e epidemiológicos dos pacientes operados.

\section{MÉTODOS}

Trata-se de um estudo retrospectivo e observacional, onde foram analisados os prontuários de pacientes de um centro cardiológico público no Estado do Pará, portadores de cardiopatia congênita com morfologia univentricular e submetidos a tratamento cirúrgico de conexão cavopulmonar parcial bidirecional (GB) como primeiro estágio.

Realizou-se uma análise dos prontuários dos pacientes submetidos a cirurgia cardíaca no período de setembro de 2011 a setembro de 2017. Sendo identificados inicialmente aqueles com morfologia univentricular que haviam sido submetidos a GB como primeiro estágio.

Foram incluídos os pacientes portadores de cardiopatia congênita com morfologia univentricular, cuja a idade variou entre 3 meses a 3 anos de vida, e abordados em condição eletiva ou de urgência hipoxêmica.

Foram excluídos os pacientes que não foram submetidos a cirurgia de conexão cavopulmonar parcial como paliação primária ou aqueles tratados inicialmente por terapêuticas endovasculares.

Foi utilizado o software BioEstat@ 5.4 para a realização da análise estatística. Foi utilizado o teste T de student para a comparação da variação da saturação periférica de oxigênio e da pressão da artéria pulmonar comparando-se a fase pré e pós-operatória. 
Foi adotado um p-valor menor que 0,05 para rejeitar a hipótese de nulidade. Esse estudo foi aprovado pelo Comitê de Ética em Pesquisa (CAAE 18888.619.8.0000.0016).

\section{RESULTADOS}

Entre setembro de 2011 a setembro de 2017 foram operados 11 pacientes com cardiopatia congênita de morfologia univentricular, submetidos à técnica de conexão cavopulmonar parcial de BG como primeiro estágio. Desse total, 6 foram meninas (54,54\%), a média de idade na admissão hospitalar foi de 10,54 meses variando de 3 meses a 2 anos e onze meses. As características anatômicas das cardiopatias foram: atresia tricúspide ( 5 casos $-45,45 \%$ ), ventrículo único tipo esquerdo ( 5 casos $-45,45 \%$ ) e ventrículo único tipo direito (1 caso - 9,09\%) (Tabela 1).

Tabela 1 - Características Anatômicas dos Cardiopatas da Amostra.

\begin{tabular}{lll}
\hline Morfologia Principal & Pacientes & $\%$ \\
\hline Atresia tricúspide IA & 2 & 18,18 \\
Atresia tricúspide IB & 3 & 27,27 \\
Ventrículo Único Tipo Esquerdo & 5 & 45,45 \\
Ventrículo Único Tipo Direito & 1 & 9,09 \\
Total & 11 & $100 \%$
\end{tabular}

Fonte: Pereira CMS, Pinheiro MF, Teixeira RK, et al (2019).

Legenda: IA: atresia tricúspide com concordância ventrículo arterial e atresia pulmonar; IB: atresia tricúspide com concordância ventrículo arterial e estenose pulmonar.

Em relação a técnica cirúrgica empregada, o acesso ao coração foi obtido através de toracotomia e esternomia mediana, seguida de dissecção e preparo da artéria pulmonar direita e veia cava superior. Foi cateterizado previamente a artéria pulmonar direita no ato intraoperatório e aferida a sua pressão sistólica. Após secção e ligadura do coto atrial, foi utilizada a técnica de anastomose com sutura contínua da porção terminal da veia cava superior, conectando-a lateralmente artéria pulmonar direita (Figura 1).

Quanto a realização de procedimentos intracardíacos associados, foi utilizado pinçamento temporário da aorta, não realizando infusão de solução cardioplégica.

Figura 1 - Aspecto intraoperatório: antes da cirurgia (1) - depois da confecção da anastomose cavo pulmonar bidirecional (2), aspecto da angiografia da artéria pulmonar após a cirurgia.

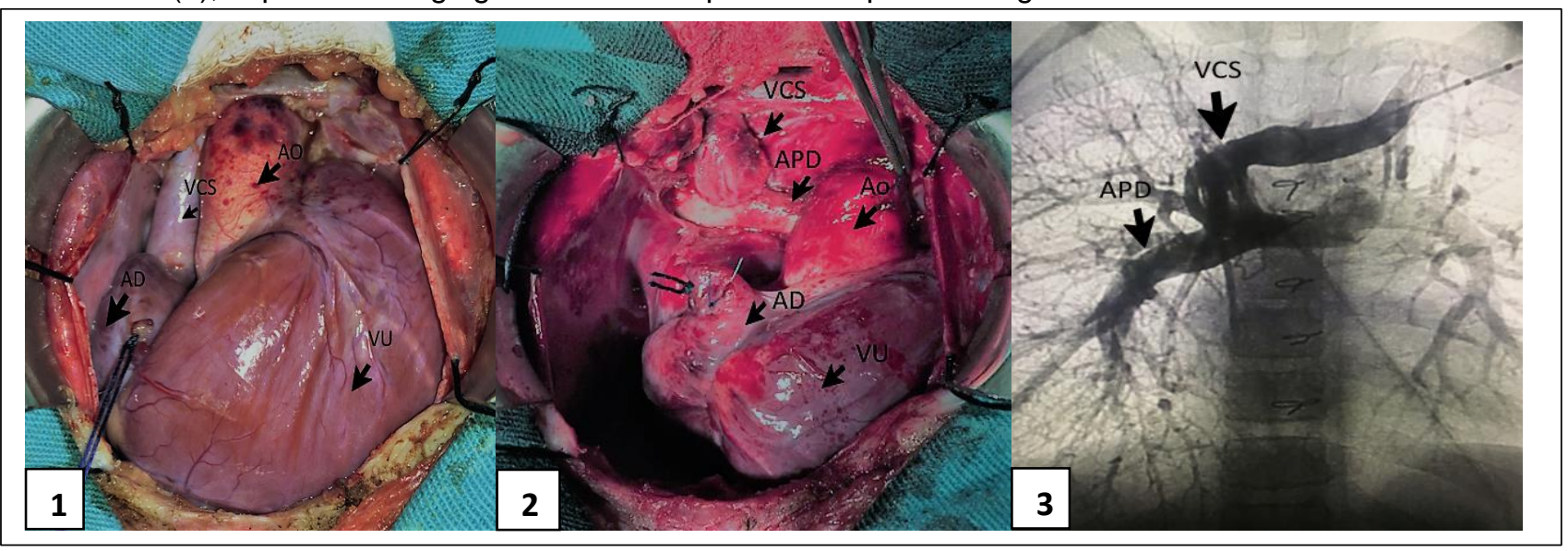

Fonte: Pereira CMS, Pinheiro MF, Teixeira RK, et al., 2019.

Legenda: VCS: veia cava superior - APD: artéria pulmonar direita - AD: átrio direito - VU: ventrículo único - AO: aorta. 
O desfecho primário avaliado foi a variação da saturação periférica de oxigênio e da pressão da artéria pulmonar comparando-se a fase pré e pós-operatória. Os demais dados avaliados foram: sexo, idade, diagnóstico da cardiopatia (obtido através de ecoDopplercardiograma transtorácico pré-operatório), caráter da cirurgia (eletiva x urgência), técnica operatória, tempo de intubação, tempo de circulação extracorpórea e anóxia, complicações operatórias, tempo de internação na unidade de terapia intensiva, permanência hospitalar e óbito.

As cardiopatias associadas ao diagnóstico principal foram: comunicação interventricular (11 casos $100,00 \%$ ), comunicação interatrial (8 casos $-72,72 \%$ ), persistência do canal arterial (4 casos - 36,36\%), estenose da artéria pulmonar ( 9 casos $-81,81 \%$ ), hipoplasia de artérias pulmonares (4 casos $-36,36 \%$ ), atresia pulmonar ( 2 casos - 18,18\%), transposição das grandes artérias ( 2 casos $-18,18 \%$ ), cortriatriatum (1 caso $-9,09 \%)$, obstrução via saída ventrículo único (1 caso - 9,09\%) e veia cava superior esquerda persistente (1 caso $-9,09 \%$ ) (Tabela 2).

Tabela 2 - Cardiopatias Congênitas Associadas.

\begin{tabular}{lcc}
\hline Cardiopatias Associadas & Quantidade & $\%$ \\
\hline C.I.A & 8 & $72,72 \%$ \\
C.I.V & 11 & $100 \%$ \\
P.C.A & 4 & $36,36 \%$ \\
Estenose pulmonar severa & 9 & $81,81 \%$ \\
Atresia Pulmonar & 2 & $18,18 \%$ \\
Cortriatriatum & 1 & $9,09 \%$ \\
Transposição das Grandes Artérias & 2 & $18,18 \%$ \\
Hipoplasia de Artérias Pulmonares & 4 & $36,36 \%$ \\
Obstrução Via Saída Ventrículo Único & 1 & $9,09 \%$ \\
Veia Cava Superior Esquerda Persistente & 1 & $9,09 \%$ \\
\hline
\end{tabular}

Fonte: Pereira CMS, Pinheiro MF, Teixeira RK, et al., 2019.

Legenda: CIA: Comunicação interatrial, CIV: comunicação interventricular, PCA (persistência do canal arterial).

Três pacientes $(27,27 \%)$ foram submetidos a cirurgia em caráter de urgência por hipóxia refratária, sendo os demais abordados em caráter eletivo. Além da realização da Conexão Cavopulmonar Bidirecional Parcial (GB) foram realizados os seguintes procedimentos associados: Bandagem de Artéria Pulmonar (8-72,72\%); Ligadura do canal arterial (6 - 54,54\%); Ampliação da comunicação interatrial (4 - 36,36\%); Ampliação da comunicação interventricular (1 - 9,09\%); Correção de Cortriatriatum (1 - 9,09\%); e Ampliação artéria pulmonar esquerda $(1-9,09 \%)$. Sete pacientes necessitaram de realização de circulação extracorpórea associada a hipotermia.

Foi mantido o fluxo pulmonar anterógrado nativo na artéria pulmonar em 8 pacientes (72,72\%), efetuando a bandagem do tronco artéria pulmonar com retalho de politetrafluoroetileno após efetivação do BG.

O tempo médio de circulação extracorpórea (CEC) foi de $51,87 \pm 18,19$ (variando de 35 a 90 minutos). 0 tempo de pinçamento aórtico variou de 4 a 10 minutos (média de 6,25 $\pm 2,63$ minutos). Houve um aumento significativo da saturação periférica de oxigênio quando comparado o pré com o pós-operatório (préoperatório: $74,46 \pm 11,81 \%$ vs pós-operatório: $88,27 \pm 3,97 \%$; $p=0,0020$ ).

Além disso, houve uma redução estatisticamente significante nos valores da pressão sistólica da artéria pulmonar (pré-operatório: $20,80 \pm 2,89 \mathrm{mmHg}$ vs pós-operatório: $17,80 \pm 1,98 \mathrm{mmHg} ; \mathrm{p}=0,0174$ ), sendo observado essa redução apenas nos pacientes submetidos a bandagem de artéria pulmonar $(n=8$; pré- 
operatório: $22,28 \pm 1,88 \mathrm{mmHg}$ vs pós-operatório: $17,85 \pm 2,41 \mathrm{mmHg} ; \mathrm{p}=0,0060)$. Nos demais casos não houve alteração nos níveis pressóricos (Gráfico 1 e 2).

Gráfico 1 - Evolução da Saturação Periférica de Oxigênio.

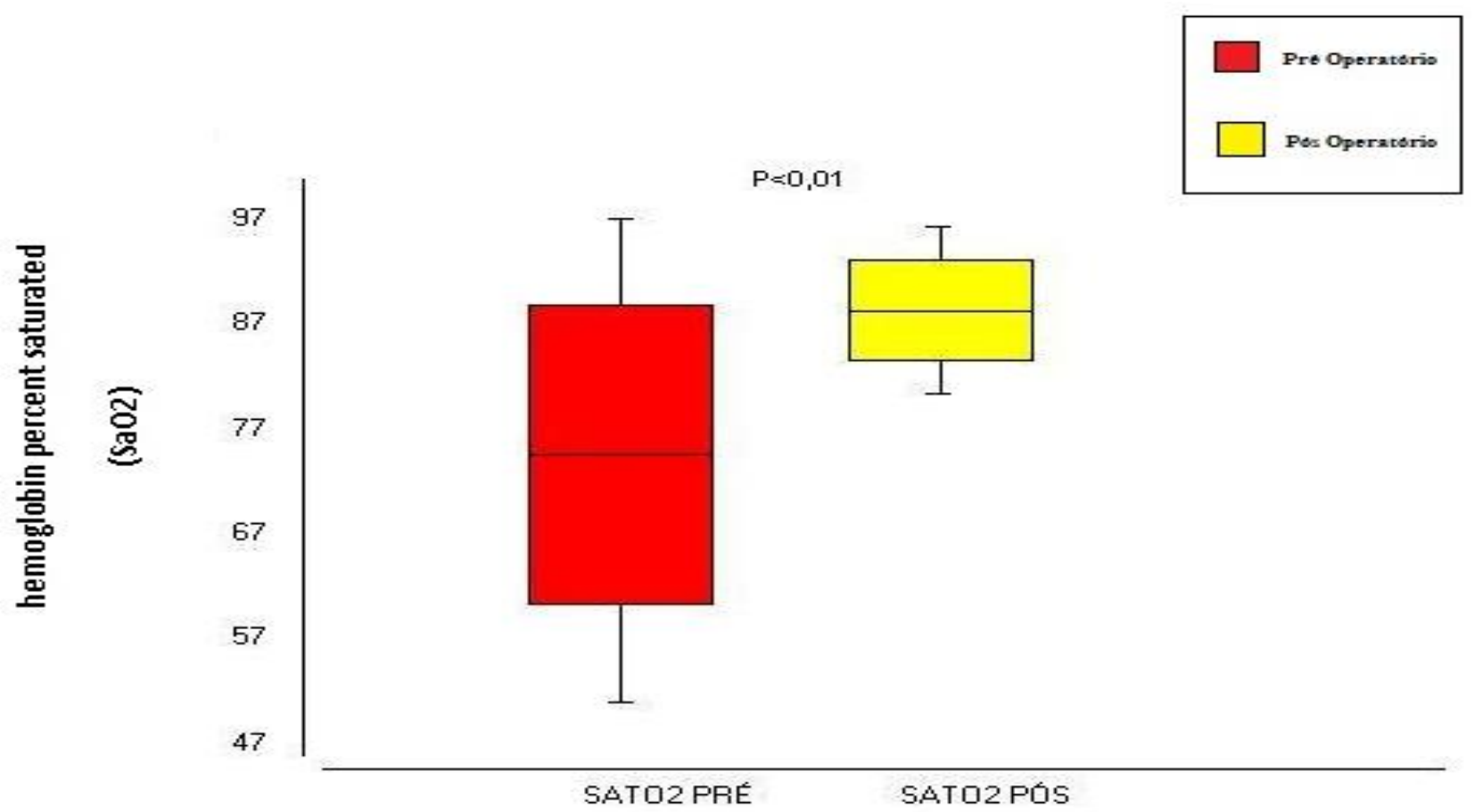

Fonte: Pereira CMS, Pinheiro MF, Teixeira RK, et al., 2019.

Legenda: Variação da saturação de oxigênio comparando-se a fase pré-operatória (vermelho) e pósoperatória (amarelo). Teste T de student e $p<0,05^{\star}$.

Gráfico 2 - Evolução da Variação da Pressão Sistólica da Artéria Pulmonar.

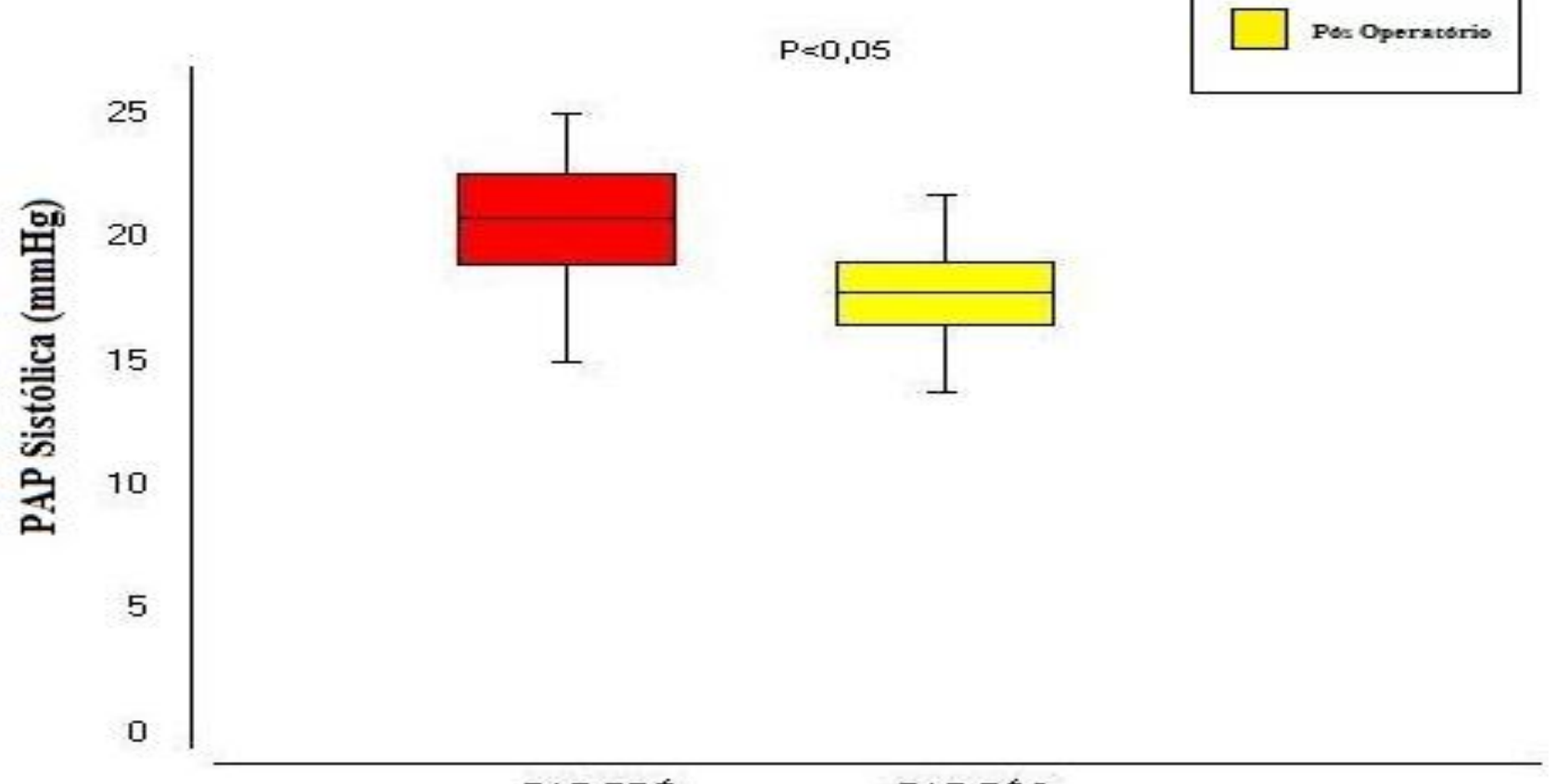


Fonte: Variação da Pressão Arterial Pulmonar comparando-se a fase pré-operatória (vermelho) e pósoperatório (amarelo). Teste T de student e $p<0,05^{*}$

Fonte: Pereira CMS, Pinheiro MF, Teixeira RK, et al (2019).

O tempo de intubação orotraqueal variou de quatro a 456 horas (média: 79,72 $\pm 137,54$ horas, mediana: $16,00 \pm 72,00$ horas), sendo os maiores valores encontrados nos pacientes que evoluíram com complicações.

Não houve óbitos na amostra analisada, entretanto ocorreram 2 episódios de paralisia frênica direita $(18,18 \%)$, sendo necessário realização de plicatura diafragmática cirúrgica; no paciente com veia cava esquerda persistente ocorreu quilotórax $(9,09 \%)$ com necessidade de pleurodese e drenagem pleural de longa permanência. Ocorreu um caso de síndrome da resposta inflamatória sistêmica pós CEC $(9,09 \%)$, sendo conduzido com suporte inotrópico e diálise peritoneal. A permanência de internação hospitalar a partir do dia da realização do procedimento cirúrgico variou de 10 a 41 dias (média de 16,9 dias (Tabela 3).

Tabela 3 - Evolução dos Pacientes na Fase Hospitalar (até 30 dias após cirurgia).

\begin{tabular}{|c|c|c|c|c|c|c|c|c|}
\hline PACIENTE & $\begin{array}{l}\text { TEMPO } \\
\text { DE CEC } \\
\text { (minutos) }\end{array}$ & $\begin{array}{l}\text { TEMPO DE } \\
\text { PINÇAMENTO } \\
\text { (minutos) }\end{array}$ & $\begin{array}{l}\text { SATO2 } \\
\text { PRÉ \% }\end{array}$ & $\begin{array}{l}\text { SATO2 } \\
\text { Pós \% }\end{array}$ & $\begin{array}{l}\text { PAP } \\
\text { PRÉ } \\
(\mathrm{mm} \mathrm{Hg})\end{array}$ & $\begin{array}{l}\text { PAP } \\
\text { PÓS } \\
(\mathrm{mmHg})\end{array}$ & $\begin{array}{l}\text { TEMPO } \\
\text { DE IOT }\end{array}$ & COMPLICACÕES \\
\hline 1 & 45 & 0 & 70 & 80 & 18 & 18 & $4 \mathrm{HS}$ & BCP \\
\hline 2 & 0 & 0 & 75 & 92 & 20 & 17 & $4 \mathrm{HS}$ & QUILOTÓRAX \\
\hline 3 & 0 & 0 & 80 & 88 & 22 & 20 & $12 \mathrm{HS}$ & \\
\hline 4 & 0 & 0 & 90 & 95 & 24 & 20 & $1 \mathrm{H}$ & \\
\hline 5 & 40 & 4 & 47 & 88 & 18 & 18 & 19 DIAS & $\begin{array}{l}\text { PARESIA } \\
\text { FRÊNICA }\end{array}$ \\
\hline 6 & 50 & 0 & 70 & 90 & * & * & $24 \mathrm{HS}$ & \\
\hline 7 & 90 & 10 & 80 & 90 & 23 & 18 & $12 \mathrm{HS}$ & \\
\hline 8 & 60 & 5 & 91 & 88 & 16 & 17 & 6 DIAS & SIRS \\
\hline 9 & 35 & 0 & 72 & 85 & 22 & 13 & 7 DIAS & $\begin{array}{l}\text { PARESIA } \\
\text { FRÊNICA }\end{array}$ \\
\hline 10 & 0 & 0 & 70 & 85 & 20 & 19 & $\begin{array}{l}16 \\
\text { HORAS }\end{array}$ & \\
\hline 11 & 55 & 6 & 75 & 90 & 25 & 18 & $12 \mathrm{HS}$ & \\
\hline
\end{tabular}

Fonte: Pereira CMS, Pinheiro MF, Teixeira RK, et al., 2019.

Legenda: *Valores não encontrados no prontuário. CEC (Circulação Extracorpórea), SATO2 PRE (Saturação de Oxigênio Pré-operatória), SATO2 POS (Saturação de Oxigênio Pós-Operatória), PAP PRE (Pressão de Artéria Pulmonar Pré Operatória); PAP PÓS (Pressão de Artéria Pulmonar Pós-Operatória); IOT (Intubação Oro traqueal); BCP (Bronco Pneumonia); SRS (Síndrome da Resposta Inflamatória Sistêmica).

O ecoDopplercardiograma pós-operatório evidenciou fluxo laminar normal na anastomose cavopulmonar em todos os pacientes, sem estenoses ou trombose. Nos pacientes em que houve ampliação da comunicação interatrial o tamanho variou de 6,7 a $13 \mathrm{~mm}$ (média: $8,52 \mathrm{~mm}$ ). A função contrátil do ventrículo único esteve preservada em todos os casos variando de $50 \%$ a $88 \%$ (média: $62,45 \%$ ). Nos casos com hipoplasia das artérias pulmonares, esta foi considerada discreta e a presença do fluxo anterógrado permitiu manutenção de saturação de oxigênio em níveis satisfatórios.

\section{DISCUSSÃO}

Desde a primeira cirurgia de conexão cavopulmonar parcial (BG) realizada em 1958 por William Glenn com a técnica unidirecional, passando a seguir para a estratégia bidirecional, as vantagens do ponto de vista hemodinâmico desta cirurgia estão bem definidas. Ocorre aumento do fluxo pulmonar sem causar sobrecarga volumétrica ventricular, levando a melhora imediata na saturação sistêmica oxigênio, além de melhorar a performance do ventrículo único devido diminuição da pré-carrega. No mais, serve como excelente paliação de preparo com intenção de conexão cavopulmonar total (HAUCK A, et al., 2017; ZAHR RA, et al., 2016; SASIKUMAR N, et al., 2014; FRIEDMAN KG, et al., 2011). 
Considerando o posicionamento geográfico da instituição onde foi realizado este estudo e sua condição de único centro em cardiopediatria cirúrgica do estado, com a peculiaridade de acolher todos os pacientes cardiopatas em idade pediátrica, eventualmente sem o devido preparo e planejamento terapêutico, ganha destaque ausência de mortalidade nesta série de casos quando comparado com achados de 2,5 a $15 \%$ em revisões de literatura (HAUCK A, et al., 2017; ZAHR RA, et al., 2016; ALSOUFI B, et al., 2018; FRIEDMAN $K G$, et al.,2011).

A idade mínima para realizar cirurgia de Glenn Bidirecional é controversa na literatura. Em estudo multicêntrico com de seguimento de longo prazo (19 anos) observou-se que a realização do Glenn antes dos 3 meses de idade aumentava significativamente a taxa de mortalidade e ainda que a distorção das artérias pulmonares e o padrão aumentado da resistência pulmonar (maior que 3 unidades Wood/m2), inerente aos lactentes jovens, foram considerados fatores de risco para o aumento de mortalidade (NICOLAS RT, et al.,2005).

Em contrapartida, outra análise abrangendo grupo expressivo de pacientes (169 casos), avaliaram comparativamente a cirurgia de Glenn bidirecional realizada em idade precoce (abaixo de 3 meses) com grupo etário superior. O tempo de permanência na UTI, tempo de ventilação mecânica e período de internação hospitalar foram maiores quando comparados ao grupo menor que 3 meses de idade. Entretanto a mortalidade precoce e tardia, bem como o tempo para realização da cirurgia de Fontan foram semelhantes entre os grupos (PETRUCCI O, et al., 2010).

No presente estudo, A idade mínima dos pacientes operados foi de 3 meses, correspondendo a $18 \%$ da amostra (2 pacientes). O tempo de intubação foi superior quando comparado ao grupo com faixa etária menor que 3 meses. Todavia relaciona-se este fato a ocorrência de paralisia frênica, pois esta aumentou a média de tempo de ventilação mecânica de 1,1 dias para 13 dias. Preconiza-se a plicatura diafragmática precoce em crianças abaixo de dois anos, pela alta morbidade da impossibilidade de extubação ventilatória (WATANABE T, et al., 1987).

É recomendável manutenção de baixas pressões na circulação pulmonar em associação com o BG para que não ocorra síndrome de veia cava superior, trombose venosa e falência da técnica (HAUCK A, et al., 2017; ZAHR RA, et al., 2016; ALSOUFI B, et al.,2018; AGARWAL A, et al., 2018). Fluxo sanguíneo pulmonar insuficiente e sem hipertensão arterial pulmonar, são condições indispensáveis para admissibilidade da técnica do BG (HAUCK A, et al., 2017; ZAHR RA, et al., 2016; PETRUCI O, et al., 2010; FRIEDMAN KG, et al.,2011). Há controvérsias na literatura acerca da manutenção do fluxo pulmonar anterógrado na ocasião da cirurgia de BG. Em análise de grupo de 246 pacientes submetidos a cirurgia de Glenn bidirecional com fluxo pulmonar anterógrado e sua capacidade de utilização como paliação temporária ou definitiva em patologias de coração univentricular, observaram que esta estratégia pode ser excelente paliação temporária ou como terapia definitiva em casos de doentes em condições clínicas não ideais (CALVARUSO DF, et al., 2008).

Outro estudo que avaliou a repercussão do fluxo pulmonar adicional mantido em pacientes submetidos ao BG, considerou valor de pressão de artéria pulmonar ideal como $18 \mathrm{mmHg}$ realizando bandagem em tronco de artéria pulmonar ou limitação de fluxo no shunt sistêmico-pulmonar caso ficasse acima do valor esperado. Neste sentido concluíram que o fluxo pulmonar adicional permitiu melhor desenvolvimento do tamanho das artérias pulmonares, sem sobrecarga de volume e beneficiando a progressão para a cirurgia de Fontan (SUZUKI Y, et al., 2010).

Em contrapartida, observou-se que a preservação do fluxo pulmonar anterógrado não conferiu aumento relevante no diâmetro da artéria pulmonar no seguimento de longo prazo. Além disso, não proporcionou benefício adicional na evolução clínica de pacientes submetidos à cirurgia de Glenn bidirecional e Fontan (GRAY RG, et al., 2007; ABDULLAH AA, 2015). Observou-se ainda que a ausência de fluxo pulmonar adicional não comprometeu a evolução favorável dos pacientes submetidos a cirurgia de BG mesmo em fase precoce (CLEZIOU J, et al., 2008).

A cirurgia de BG realizada sem circulação extracorpórea é segura, além de evitar os efeitos indesejáveis do desvio cardiopulmonar, sugerindo ainda ser mais viável economicamente (HUSSAIN ST, et al., 2007). 
Estudos comparando a cirurgia de BG sem utilização de CEC com pacientes operados com desvio cardiopulmonar, observaram que ambas as abordagens são seguras em relação à morbidade e mortalidade. Entretanto a utilização de ultrafiltração modificada associada à CEC mostrou-se ferramenta fundamental no resultado desta cirurgia quando o desvio é utilizado (CROTI UA, et al., 2007). Neste estudo, o tempo de hospitalização, tempo de extubação, incidência de complicações neurológicas e derrame pleural foram semelhantes entre os dois grupos. Outra análise mostrou ser possível realizar técnica de BG sem CEC com minitoracotomia direita, sem realizar a clássica esternomia (GUIDA M, et al., 2015).

No atual estudo a realização da cirurgia de BG sem a utilização de circulação extracorpórea conferiu a estes doentes menores tempos de intubação orotraqueal, quando comparados aos pacientes em que a realização do desvio cardiopulmonar foi necessária. A amostra deste estudo não permitiu análise comparativa estatisticamente significante entre os grupos com e sem CEC, sobretudo devido o número de casos sem desvio cardiopulmonar ter sido pequeno (4 pacientes).

Merece destaque o fato de que neste estudo retrospectivo de série de casos não foram incluídos pacientes submetidos a procedimentos paliativos prévios ao BG, excluíram-se habitualmente circunstâncias anatômicas com elevada mortalidade, tais como prematuridade, baixo peso, síndrome do coração esquerdo hipoplásico, síndromes genéticas com anomalias extra cardíacas, bem como defeitos do arco aórtico. Portanto o grupo de coorte selecionado no presente estudo interferiu na avaliação de mortalidade, impondo eventual viés, considerando-se sobretudo que grupos de grave associações patológicas necessitam de cirurgia emergencial e cuja mortalidade pode ultrapassar 10\% (ALSOUFI B, et al., 2018).

Outros fatores anatômicos foram descritos como relacionados a maior mortalidade, a qual variaria de 5 a $15 \%$, tais como como síndrome heterotáxica, drenagem anômala de veias pulmonares, anomalias anatômicas em artérias pulmonares, hipertensão arterial pulmonar e ventrículo direito sistêmico (MANUEL V, et al., 2019; TANOUE Y, et al., 2007).

Os pacientes desta amostra não tinham na sua apresentação clínica os fatores de mau prognóstico descritos anteriormente, o que confere a estes, candidatos cirúrgicos com melhor prognóstico a curto prazo segundo a literatura pesquisada. $O$ que contribuiu para ausência de mortalidade encontrada na presente amostra no período estudado.

\section{CONCLUSÃO}

A análise das crianças tratadas no presente estudo não contempla a fase de evolução a médio e longo prazo. Não obstante, mesmo sem realizar análise comparativa das cirurgias de GB com grupo controle de pacientes submetidos à cirurgia de shunt sistêmico pulmonar, constatou-se bom nível de saturação pósoperatória com excelente desempenho hemodinâmico. Após correlacionar os resultados desta série de casos deste com as publicações em grandes centros mundiais, nota-se que houve benefício em realizar o BG como primeiro estágio, entretanto supõe-se que as condições hemodinâmicas relacionadas a cardiopatia da amostra avaliada permitiram estabelecer a tática cirúrgica, sendo eventualmente esperado como conduta mais recomendada não confeccionar shunt sistêmico pulmonar nestes pacientes. Os resultados deste estudo sugerem que a confecção da anastomose cavopulmonar parcial (BG) como paliação primária no tratamento do coração univentricular tem resultados imediatos satisfatórios quando realizada acima dos três meses de idade e em associação com estenose ou atresia pulmonar.

\section{REFERÊNCIAS}

1. HAUCK A, et al. The Pulmonary Circulation in the Single Ventricle Patient. Children, 2017; 4-71

2. ZAHR RA, et al. Half Century's Experience with the Superior Cavopulmonary (Classic Glenn) Shunt. Annals of Thoracic Surgery 2016; 101:177-82.

3.SASIKUMAR N, et al. Outcome of Blalock-Taussig shunts in currrent era: A single center experience. Congenital Heart Disease. 2017;1-7

4.SASIKUMAR N, et al. Pulmonary artery banding for univentricualr heart beyound the neonatal period. Asian Cardiovasc Thorac Ann. 2014; 22: 660-6 
5.ALSOUFI B, et al. Factors Associated with Interstage Mortality Following Neonatal Single Ventricle Palliation. World Journal for Pediatric and Congenital Heart Surgery. 2018; 9:616-623

6.AGARWAL A, et al. Incidence and Management of Thrombotic and Thromboembolic Complications Following the Superior Cavopulmonary Anastomosis Procedure: A Literature Review. Clinical and Applied Thrombosis/Hemostasis 2018; 24:405-415

7.NICOLAS RT, et al. Early outcome after Glenn shunt and Fontan palliation and the impact of operation during viral respiratory season: analysis of a 19-year multi-institutional experience. Ann Thorac Surg 2005; 79:613-7

8.PETRUCCI O, et al. Outcomes of The Bidirecional Glenn procedure in Patients Less Than 3 Months of Age. Journal of Thoracic Cardiovascular Surgery 2010; 139:562-568.

9.WATANABE T, et al. Phrenic nerve paralysis after pediatric cardiac surgery retrospective study of $125 \mathrm{cases}$. J Thorac Cardiovsc Surg 1987; 94:383-8

10.CALVARUSO DF, et al. Bidirectional Glenn and antegrade pulmonary blood flow: temporary or definitive palliation? Ann Thorac Surg. 2008; 85:1389-95.

11.SUZUKI Y, et al. Bidirectional Cavopulmonay Shunt with Additional Pulmonary Blood Flow. Asian Cardiovasc Thorac Ann 2010; 18:135-140

12.GRAY RG, et al. Persistent antegrade pulmonary blood flow post-Glenn does not alter early post-Fontan outcomes in single-ventricle patients. Ann Thorac Surg. 2007; 84: 888-93

13.ABDULLAH AA. Bidirecional Glenn With Addictional Pulmonary Blood Flow: Systematic Review and Evidance-Based Recommendations. Journal Card Surg 2015; 30:724-730

14.CLEZIOU J, et al. Bidirectional cavopulmonary connection without additional pulmonary blood flow in patients below the age of 6 months. European Journal of Cardio-Thoracic Surgery. 2008; 34: 556-62.

15.HUSSAIN ST, et al. The bidirectional cavopulmonary (Glenn) shunt without cardiopulmonary bypass: is it a safe option? Interact Cardiovasc Thorac Surg. 2007; 6: 77-82

16.CROTI UA, et al. Should the Bidirectional Glenn Operation be performed with or without cardiopulmonary bypass? Brazilian Journal of Cardiovascular Surgery. 2004; 19: 274-279.

17.GUIDA M, et al. Off-pump bidirectional Glenn through right anterior thoracotomy. Braz J Cardiovasc Surg. 2015; 30:497-500

18.MANUEL V, et al. Single Ventricle Palliation in a Developing Sub-Saharan African Country: What Should be Improved? World Journal for Pediatric and Congenital Heart Surgery. 2019; 10:164-170.

19.FRIEDMAN KG, et al. Risk factors for failed staged palliation after bidirectional Glenn in infants who have undergone stage one palliation. Eur Journal Cardiothorac Surg. 2011; 40: 1000-1006.

20.TANOUE Y, et al. Three Hundred and Thirty-three experiences With the Bidirecional Glenn Procedure in a Single Institute. Interact Cardiovascular Thoracic Surgery 2007; 6: 97-101.

21. PINTO VC, et al., Epidemiology of congenital heart disease in Brazil. Braz J Cardiovasc Surg 2015;30(2):219-24.

22. VAN DER LINDE D, et al., Birth prevalence of congenital heart disease worldwide. A systematic review and metaanalysis. J Am Coll Cardiol. 2011;58(21):2241-7.

23. GO AS, et al., American Heart Association Statistics Committee and Stroke Statistics Subcommittee. Heart disease and stroke statistics-2013 update: a report from the American Heart Association. Circulation. 2013;127. 